\title{
Research on Interactive Control of Electrolytic Aluminum Load and Wind Power Output
}

\author{
Ge Simin ${ }^{1}$, Yu Kun ${ }^{1,2}$ \\ ${ }^{1}$ Hohai University, College of Energy and Electrical Engineering, 210098 Nanjing, China \\ ${ }^{2}$ Jiangsu Engineering Research Center for Distribution \& Utilization and Energy Efficiency, 210098 Nanjing, China
}

\begin{abstract}
With the large-scale wind power generation connected to Gansu power grid, electrolytic aluminium load has reached a certain scale at the same time, the vast majority of electrolytic aluminium load directly connects to power grid using $330 \mathrm{kV}$ transmission line. According to the physical characteristics and historical data, the continuous adjustment characteristic of electrolytic aluminium load is analysed. Based on this characteristic, a mathematical model for the electrolytic aluminium load is established. Aiming at reducing power network loss and wind power consumption, an optimization model based on the load regulation characteristics of electrolytic aluminium is constructed, which is optimized by particle swarm optimization algorithm. Based on the case data of Gansu power grid, the optimal method based on the load regulation characteristics of electrolytic aluminium is analysed and its feasibility is verified.
\end{abstract}

\section{Instruction}

Gansu Province has lots of wind energy resources [1] which are mainly concentrated in Jiayuguan and Jiuquan area, and are away from the province's electricity load center. Further more, lots of wind power can not be consumed since the wind power consumptive ability in Jiayuguan and Jiuquan areas is very limited [2]. The wind power could be transmitted to the main power grid of Gansu province through double circuit $750 \mathrm{kV}$ transmission line in Jiuquan Hexi. However, remote delivery of large-scale active power increases the loss of power system and also reduces the voltage quality of Hexi power grid and the main power grid of Gansu province, which increase the network loss value more. In addition, due to the reverse distribution characteristic of the new energy and conventional generations [3], the power loss is huge when conventional generations are adjustable. The randomness and volatility of the new energy sources [4] may lead to a large voltage fluctuation of the transmission line, which further increase the power loss [5]. To solve this problem, paper [6] reduces the randomness of new energy output by using the coupling of different new energy sources, but it does not solve the problem of large-scale new energy consumption. Aiming at consuming the new energy, paper [7] achieves dynamic economic dispatch by optimizing the power of conventional thermal power generating units, which makes all the wind power output be consumed by power grid. However, sometimes only by coordinating with conventional power supply, the new energy output cannot be completely consumed. Paper [8] uses spot price to stimulate demand side resources to consume the wind generation output, but the analysis of demand side resources is not detailed enough.

Electrolytic aluminium industry [9-10] in Gansu Province are one of the most important industries, which has large capacity and regulation characteristic, usually use $330 / 750 \mathrm{kV}$ high voltage to supply the power directly. Based on the regulation characteristics of electrolytic aluminium load, the load cooperates with the new energy to consume as much new energy as possible and reduce the power loss. In paper [11], the characteristics of high energy load are analysed and the corresponding mathematical model is established, but there is no specific method or strategy towards the power loss reduction problem based on load features of electrolytic aluminium.

In this paper, based on the characteristic analysis of the electrolytic aluminium load, the mathematical model of electrolytic aluminium load is constructed. The interactive optimization model based on the load regulation characteristics of electrolytic aluminium is established aiming at reducing power network loss and wind power consumption. Particle swarm optimization algorithm is used to optimize the model, and the power loss reduction method based on the load regulation characteristics of electrolytic aluminium is analysed. Its feasibility is verified by a numerical example.

\section{Load characteristics and mathematical model of electrolytic aluminium}




\subsection{Continuous regulation characteristic of electrolytic aluminium load}

Rhyolite molten salt electrolysis [12] has been used in modern aluminium electrolysis industry, with powerful direct current into, electrochemical reaction happens at a high temperature on the two poles of the electrolytic tank to produce aluminium metal. In this production process, the electrolytic tank needs to be continuously supplied with direct current, and the size of electrolytic aluminium load current can be regulated by controlling the conduction angle of thermistor. The entire production process requires a constant current. If the direct current fluctuates largely, it will significantly affect the stability of the operation state (especially thermal equilibrium) of the electrolytic tank, and will interfere normal control process of aluminium electrolysis, thus the technical and economic indicators of electrolytic aluminium production will be influenced. Therefore, when the electrolytic aluminium load is regulated, the load production reduction needs to be in the scope of the electrolytic tank, and the load current of the electrolytic aluminium can be adjusted by controlling the conduction angle of the controllable silicon.

According to the above analysis, under normal circumstances, the fluctuation of electrolytic aluminium load capacity is small, the load curve is relatively smooth, as shown in Fig. 1. Since the electrolytic aluminium load often has a number of feeders, each feeder is connected to a rectifier unit, and each unit can be adjusted within a certain range. Through the analysis of historical data, the adjustment range of electrolytic aluminium load is $10 \% \sim 10 \%$.

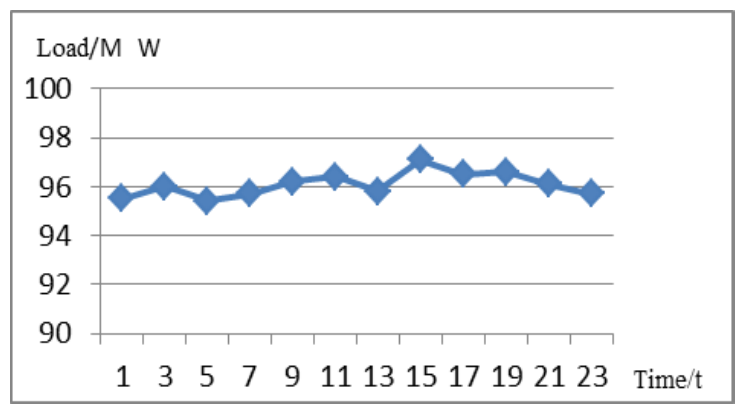

Figure 1. Typical daily load curve of electrolytic aluminium.

\subsection{Mathematical model of continuous adjustable for electrolytic aluminium load}

In the adjustable state of electrolytic aluminium load, when the load began to participate in the regulation, the load will change from load rating according to the ramp rate, and stop by the set value. When the set value is lower than the current value, the ramp rate is called the load reduction ramp rate. When the set value is lower than the current value, the ramp rate is called the load increase ramp rate. The ramp rate of aluminium load differs among different units.

In order to maintain the normal production of electrolytic aluminium load, the electrolytic aluminium production amount should fluctuate within the allowable range, and the set value of load should be in the allowable range as well. Use a single regulation as an example, if the load is adjusted from rating to a set value and then return to the rating, the typical electrolytic aluminium load regulation curve is shown in Fig. 2.

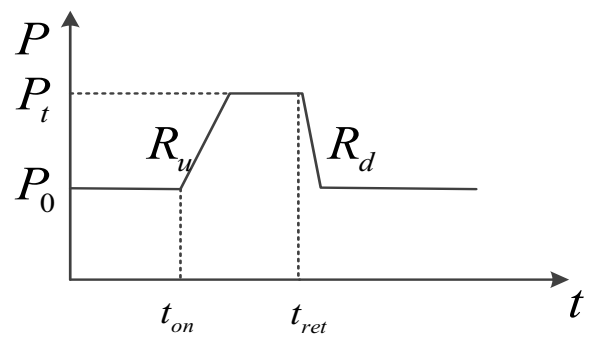

Figure 2. Typical load regulation curve of electrolytic aluminium.

The mathematical model of the load regulation characteristics of electrolytic aluminium can be established by the above regulation curve:

$$
P=\left\{\begin{array}{l}
P_{0}, t \leq t_{\text {on }} \\
P_{0}+R_{u}\left(t-t_{\text {on }}\right), t_{\text {on }}<t \leq t_{\text {on }}+\frac{P_{t}-P_{0}}{R_{u}} \\
P_{t}, t_{\text {on }}+\frac{P_{t}-P_{0}}{R_{u}}<t \leq t_{\text {ret }} \\
p_{t}-R_{d}\left(t-t_{r e t}\right), t_{r e t}<t \leq t_{r e t}+\frac{P_{t}-P_{0}}{R_{d}} \\
P_{0}, t>t_{r e t}+\frac{P_{t}-P_{0}}{R_{d}} \\
P_{\min } \leq P \leq P_{\max } \\
c_{i}=\sum_{i=1}^{T} \frac{p_{i, t}}{\eta_{i}} \\
c_{\min } \leq c_{i} \leq c_{\max }
\end{array}\right.
$$

where $P_{0}$ represents the capacity rating of the load or the value of the load capacity before adjustment, $t_{\text {on }}$ represents trigger time of load regulation, $R_{u}$ represents the load increase ramp rate, $t_{\text {ret }}$ represents the return time or the next time adjustment, $R_{d}$ represents the load reduction ramp rate, $P_{\min }$ represents the minimum value of the load regulation range, $P_{\max }$ represents the maximum range of load regulation, $c_{i}$ represents load production amount, $p_{i, t}$ represents the active power consumption of the load at the time of $t$, $\eta_{i}$ represents the unit product power consumption, $c_{\min }$ represents the minimum value of the load production amount range, $c_{\max }$ represents the maximum value of the load production amount range.

When the load needs to be adjusted down to the set value, the mathematical model can be adjusted on the basis of the formula (1) as follows. 


$$
P=\left\{\begin{array}{l}
P_{0}, t \leq t_{\text {on }} \\
P_{0}-R_{d}\left(t-t_{\text {on }}\right), t_{\text {on }}<t \leq t_{\text {on }}+\frac{P_{0}-P_{t}}{R_{d}} \\
P_{t}, t_{o n}+\frac{P_{0}-P_{t}}{R_{d}}<t \leq t_{r e t} \\
p_{t}+R_{u}\left(t-t_{r e t}\right), t_{r e t}<t \leq t_{r e t}+\frac{P_{0}-P_{t}}{R_{u}} \\
P_{0}, t>t_{r e t}+\frac{P_{t}-P_{0}}{R_{d}}
\end{array}\right.
$$

\section{Optimization of network loss and wind power consumption based on the regulation characteristics of electrolytic aluminium load}

From analysis above, electrolytic aluminium load has adjustable characteristics, but constraints of load need to be considered during the regulation. When the electrolytic aluminium load is involved in the process of power loss reduction, it should be cooperated with conventional generation to achieve active power balance.

\subsection{A network loss optimization model considering the load regulation characteristics of electrolytic aluminium}

\subsection{1 objective function}

Usually, daily load curves are similar, so optimization result of one chosen day is representative. The optimization sets the total loss on the power grid to be minimum as the goal, including line loss and transformer loss.

$$
\min f \sum_{t=1}^{T}\left(\sum_{i=1}^{N} \frac{P_{i}^{2}+Q_{i}^{2}}{U_{i}^{2}} R_{L i}+\sum_{j=1}^{M} \frac{P_{j}^{2}+Q_{j}^{2}}{U_{j}^{2}} R_{T j}\right)
$$

where $P_{i}, Q_{i}, U_{i}$ each represents the active power, reactive power and voltage at the first end of the branch $i$, and $R_{L i}$ is line impedance. $P_{j}, Q_{j}, U_{j}$ each represents the active power, reactive power and voltage at the first end of the branch $j$, and $R_{T j}$ is transformer impedance. $N$ is the total number of lines, $M$ is the total number of transformers, $T$ is the total length of time.

\subsection{2 constraint condition}

Firstly, the active power balance of power system is considered:

$$
\sum P_{G}=\sum P_{L}+\sum P_{L O S S}
$$

where $\sum P_{G}, \sum P_{L}, \sum P_{L O S S}$ each represents the total power output of the system, the total load and total transmission loss. The total generating capacity includes the wind generation output and conventional power output.

Secondly, the electrolytic aluminium load constraints are considered, the adjustment process must be in electrolytic aluminium adjustable range, including the limit value of load:

$$
P_{L \text { min }} \leq P_{L} \leq P_{L \text { max }}
$$

where $P_{L \min }$ represents the minimum value of the load regulation range, $P_{L \max }$ represents the maximum value of the load regulation range.

Load ramp rate constraint:

$$
\Delta P_{\max d o w n} \leq P_{a d j}(t)-P_{a d j}(t-1) \leq \Delta P_{\max u p}
$$

where $\Delta P_{\text {max down }}$ represents the maximum decreasing load variation, $\Delta P_{\max \text { up }}$ represents the maximum increasing load variation, $P_{a d j}(t)$ represents load active power of time $t, P_{a d j}(t-1)$ represents load active power of time $(t-1)$.

Since the normal production should not be affected during the regulation, it is ensured by the order production constraints:

$$
c_{\min } \leq c_{i} \leq c_{\max }
$$

where $c_{\min }$ represents the minimum load production amount, $c_{\max }$ represents the maximum load production amount.

Output constraint of conventional power supply:

$$
P_{G \text { min }} \leq P_{G} \leq P_{G \max }
$$

where $P_{G \min }$ represents the minimum value of the conventional power regulation range, $P_{G \max }$ represents the maximum value of the conventional power regulation range.

\subsection{An optimization algorithm considering the regulation characteristics of electrolytic aluminium load}

According to the analysis above, the network loss optimization problem that considers the regulation characteristic of electrolytic aluminium load is a typical constrained nonlinear programming problem, using particle swarm algorithm ${ }^{[13]}$ to solve and the specific steps of the algorithm are as follows..

(1) Firstly, encode the active power of electrolytic aluminium load, wind power generation output and conventional generation output, code value of electrolytic aluminium load is its active power in the power grid and code value of conventional generation and wind generation are their output. The range of code value is ensured by the given load and generation.

(2) The number of electrolytic aluminium load, wind power generation and conventional generation decides the dimension $\mathrm{m}$ of particle swarm. Random 
function is used to encode each particle. If the number of particles is $n$, then there will be an $n^{*} m$ matrix variables, which represents the population in particle swarm algorithm. At the same time, random function is used to initialize the speed of each particle. Set historic individual optimal position zbest as current location, and set the best of group as current gbest.

(3) The population that is composed of electrolytic aluminium load active power, wind generation output and conventional generation output is the independent variable, while network loss value is the dependent variable. Set the network loss value as the fitness function, through the power flow calculation, the value of fitness function of each particle can be achieved.

(4) If the current value of fitness function of the particle is better than its historical optimal value, then the historical optimum will be replaced by the current position.

(5) If the historical optimal of the particle is better than its global optimal, then the global optimal will be replaced by its historical optimal.

(6) Update the speed and position of each particle according to the following formula.

$$
\begin{gathered}
v_{i}(k+1)=w v_{i}(k)+c_{1} r_{1}\left(p_{i}-x_{i}(k)\right)+c_{2} r_{2}\left(p_{g}-x_{i}(k)\right) \\
x_{i}(k+1)=x_{i}(k)+v_{i}(k+1)
\end{gathered}
$$

where $p_{i}$ represents individual extremum, $p_{g}$ represents the extremum of the group, $w, c_{1}, c_{2}$ are constants, $v_{i}(k), v_{i}(k+1)$ each represents the current speed of the particle $i$ in step $k$ and step $k+1$ during the iteration, $x_{i}(k), x_{i}(k+1)$ each represents the current position of the particle $i$ in step $k$ and step $k+1$ during the iteration.

(7) Iteration number plus 1 . if the condition of end is not reached, then go to the third step, otherwise output the result and end.

\section{Example analysis}

Based on the data of Gansu power grid, an example is constructed as shown in Fig. 3 to analysis the method of network loss reduction and wind power consumption involving electrolytic aluminium load. The validity of the proposed method is verified. There are 2 wind generations, wind generation unit 1 has the active power of $60 \mathrm{MW}$, and is connected to the net through node 1 grid, wind generation unit 2 has the active power of $70 \mathrm{MW}$, and is connected to the net through node 9. Node 6 and node 11 are both conventional generations, and node 7 is connected with electrolytic aluminium load, whose active power is $310 \mathrm{MW}$.

As the result of simulation, network loss reduces from $277 \mathrm{MW}$ to $268 \mathrm{MW}$, the online capacity of wind generation unit1 increases from $60 \mathrm{MW}$ to $67 \mathrm{MW}$ and the online capacity of wind generation unit 2 increases from $70 \mathrm{MW}$ to $75 \mathrm{MW}$ when conventional generation participates during the process.

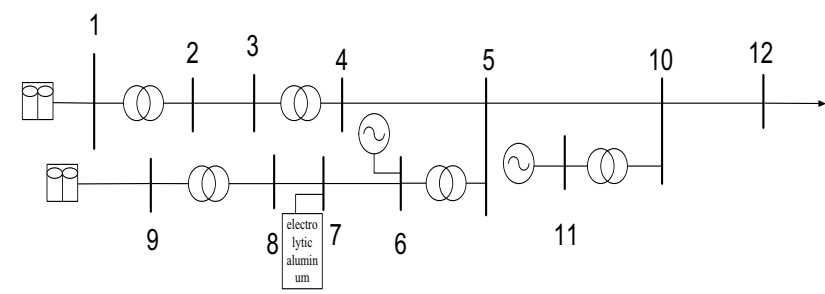

Figure 3. Simulation example wiring diagram.

On the basis of conventional generation participating in regulation, the network loss can be reduced to $256 \mathrm{MW}$ when considering the continuous adjustable characteristic of electrolytic aluminium load. The percentage of the reduction of network loss is $7.6 \%$, which is more than twice as high as the regulation using conventional generation. And the online active power of wind generation raises more as well, unit1 increases $20 \%$ and unit2 increases $17 \%$.

In other words, the network loss can be reduced and the consumption of wind generation can be increased in some degree by regulating conventional generation. And if the continuous regulation characteristic of electrolytic aluminium load is considered, the change of both network loss and wind power consumption would be more significantly.

\section{Conclusions}

In this paper, according the actual background of Gansu power grid, based on the analysis of the electrolytic aluminium load, the mathematical model of electrolytic aluminium load is set up. Aiming at reducing network loss, the optimization model of power network loss reduction based on the load regulation characteristics of electrolytic aluminium is established. Due to the characteristics of the model, particle swarm optimization algorithm is used to optimize the model. The example is constructed based on the data of Gansu power grid, and the power loss reduction method based on the load regulation characteristics of electrolytic aluminium is analysed. The result shows that regulate electrolytic aluminium load and conventional generations at the same time can significantly reduce the network loss.

\section{Acknowledgements}

This research is supported by the National Development and Reform Commission (the research, development and industrialization for integrated management of smart distribution grid \& utilization and enhancing energy efficiency technology based on internet of things).

\section{References}

1. Xiao Chuangying, Wang Ningbo, Zhi Jing, et al, Automation of Electric Power Systems, 64 (2010)

2. Liu Chang, Wu Hao, Gao Changzheng, et al, Power System Protection and Control, 61 (2014) 
3. Cui Hong, Xia Chengjun, Luo Zongjie, et al, Electric Safety, 54 (2009)

4. Zhen Xin, Yang Dezhou, Wang Liping, et al, Power System Protection and Control, 39 (2011)

5. Liu Dewei, Huang Yuehui, Wang Weisheng, et al, Automation of Electric Power Systems, 77 (2011)

6. Luo Qing, Chao Qin, Wang Yibo, et al, Electric Power Automation Equipment, 42 (2014)

7. Zhou Wei, Peng Yu, Sun Hui, et al, Proceedings of CSEE, 13 (2009)
8. Liu Xiaocong, Wang Beibei, Li Yang, et al, Power System Technology, 2955 (2014)

9. Qiu Zhuxian, Engineering Science, 41 (2003)

10. Lu Yufei, Yuannan Metallurgy, 58 (2004)

11. Chen Runze, Sun Hongbin, Jin Hongyang, et al, Automation of Electric Power Systems, 168 (2015)

12. Feng Naixiang, Peng Jianping, Wang Yaowu, et al, Journal of Material and Metallurgy, 1 (2010)

13. Chen Jianhua, Li Xianyun, Deng Donghua, et al, Power System Technology, 77 (2007) 